\title{
Improved treatment satisfaction in patients with type 2 diabetes treated with once-weekly semaglutide in the SUSTAIN trials
}

Johan Jendle MD, Andreas L. Birkenfeld MD, William H. Polonsky PhD, Robert Silver MD, Kari Uusinarkaus MD, Thomas Hansen PhD, Jonas Håkan-Bloch MSc, Sayeh Tadayon MD, Melanie J. Davies MD

\section{Video Abstract}

Keywords: SUSTAIN, glucose, diabetes, type 2 diabetes, glycemic targets, semaglutide, GLP-1 RA, glucagon-like peptide-1 receptor agonist, weight loss, phase 3 trial, HbA1c, sitagliptin, exenatide extended release, insulin glargine, dulaglutide, Diabetes Treatment Satisfaction Questionnaire, DTSQ, placebo, treatment satisfaction, Diabetes Obesity and Metabolism

Posted Date: October 10th, 2019

DOI: https://doi.org/10.21203/rs.2.16123/v1

License: (9) This work is licensed under a Creative Commons Attribution 4.0 International License. Read Full License 


\section{Abstract}

Despite the availability of effective and safe glucose-lowering agents, many patients with type 2 diabetes do not achieve glycemic targets. This finding suggests that when it comes to deciding on treatment options, efficacy and safety are only part of the equation; patients' preferences, needs, and values should also guide decision-making. In this vein, researchers explored the attitudes of patients with inadequately controlled type 2 diabetes towards semaglutide. Semaglutide is an injectable, once-weekly glucagon-like peptide-1 receptor agonist, or GLP-1 RA. Growing research has shown semaglutide to be safe and effective for controlling glucose levels and to induce weight loss in people with type 2 diabetes. In a subset of the phase 3 clinical trials collectively known as SUSTAIN, once-weekly subcutaneous injections of semaglutide consistently provided superior $\mathrm{HbA} 1 \mathrm{c}$ and body-weight reductions versus placebo or active comparators, namely, sitagliptin, exenatide extended release, insulin glargine, and dulaglutide. To understand patients' satisfaction with each treatment, the research team analyzed patient responses to the Diabetes Treatment Satisfaction Questionnaire gathered in SUSTAIN trials 1 to 5 and 7. Those responses showed that semaglutide was associated with significantly greater overall treatment satisfaction compared with the other treatment options or placebo added on to basal insulin. At worst, patients' reported satisfaction with semaglutide was on par with that reported for dulaglutide. Post hoc analyses indicate that glycemic efficiency and weight loss may play a role in determining the level of treatment satisfaction. The change in overall treatment satisfaction from baseline was generally greater, although not always statistically significant, in patients who lost more than $5 \%$ body weight or reached $\mathrm{Hb} 1 \mathrm{Ac}$ levels below $7 \%$ than in those who did not. Additionally, the team found no compelling evidence that gastrointestinal adverse events -the type of side effect most commonly reported for GLP-1 RAs-had a significant, sustained effect on patient-perceived treatment satisfaction. Some caution is required in interpreting these findings, as the diabetes-specific questionnaire used does not reflect the characteristics specific to GLP-1 RAs. Moreover, any subjective, patient-reported outcomes is subject to potential bias. Nevertheless, the findings are instructive. The clinical benefits of once-weekly semaglutide versus placebo and active comparators seen across the SUSTAIN trial program in people with type 2 diabetes are accompanied by significantly greater or similar improvements in overall treatment satisfaction. The results also advocate treatment satisfaction, in addition to efficacy and safety, as a factor to consider when selecting glucose-lowering therapy. 